\title{
Educación Intercultural Bilingüe en Ecuador: Una revisión conceptual
}

\section{Ecuadorian Intercultural Education: a conceptual review}

Guadalupe Vernimmen Aguirre es profesora titular de la Universidad de Guayaquil (Ecuador) (guadalupe.vernimmena@ug.edu.ec) (1D (https://orcid.org/0000-0003-2942-9022)

Recibido: 2018-02-14 / Revisado: 2019-04-06 / Aceptado: 2019-05-16 / Publicado: 2019-07-01

\section{Resumen}

El artículo explora los desafíos de la Educación Intercultural Bilingüe (EIB) en Ecuador. Para ello, se analizan los antecedentes teóricos e históricos del proceso de institucionalización de la EIB. Toma en cuenta las resistencias de los movimientos indígenas por educarse en una lengua ancestral desde sus inicios hasta llegar a la institucionalización del Sistema de Educación Intercultural Bilingüe (SEIB) en el año 1988. El problema que se plantea el presente estudio es: ¿cuáles son las principales conceptualizaciones de la interculturalidad que permiten reflexionar alrededor de la EIB en Ecuador a favor del desarrollo y las políticas públicas? Como objetivo general se analiza el devenir de la EIB, los conceptos y marcos legales que la sitúan. El ensayo se adhiere al estudio crítico de la EIB en el marco de las relaciones entre culturas y cómo estas podrían fortalecerse o simplemente intercambiarse. Se presenta como hipótesis preliminar que en el país ecuatoriano se avala una educación más multicultural que intercultural y esto incide en el desarrollo social, por lo tanto, es fundamental la implementación de políticas públicas culturales que también impulsen la interculturalidad como proyecto político y ético en Ecuador.

Descriptores: Interculturalidad, políticas públicas, desarrollo social, cultura, educación.

\begin{abstract}
The article explores the challenges of Intercultural Bilingual Education in Ecuador (EIB). For this, it reviews the theorical background and the history of the institutionalization of the EIB. It considers the history of the struggles and resistances found by indigenous movements for educating themselves in an ancestral language from its beginnings up to the institutionalization of the Bilingual Intercultural Education System (SEIB) in 1988. the problem of this study is: which are the principal conceptualitation of interculturality that allows us to reflect around the EIB in Ecuador, for developing and public policies. As a general objective there is an analysis of the EIB in the order of the relationship between cultures and how these can be strengthened or have as simply interchange. The preliminary hypothesis is that the Ecuadorian country it is allowed a more multicultural education rather than the intercultural education and that affects local developing, son it's a fundamental to implement cultural public policies that also allows the interculturality as a political an ethic project in Ecuador.

Keywords: Interculturality, cultural policy, indigenous, communication, cultural development, decolonization.
\end{abstract}

Forma sugerida de citar: Vernimmen Aguirre, G. (2019). Educación Intercultural Bilingüe en Ecuador: Una revisión conceptual. Alteridad, 14(2), 162-171. https://doi.org/10.17163/alt.v14n2.2019.01 


\section{Introducción}

Es neurálgico revisar las consecuencias del capitalismo colonial en América Latina. Las condiciones históricas que permitieron que lenguas como el kichwa sean consideradas sin valor, pero que al mismo tiempo hayan sido estratégicamente utilizadas por los colonizadores españoles en distintos momentos para dominar. Los llegados a América aprendieron el idioma para comunicarse con los indígenas y enseñar la religión cristiana imperante (mientras se desprestigiaban las creencias ancestrales originales), posteriormente, durante la modernidad se recibió apoyo internacional para impulsar la educación de los pueblos indígenas y lo hacían en la lengua indígena porque desde la lucha de los movimientos indígenas imperaba la necesidad de ser incluidos en los proyectos de educación, lo que posteriormente dio lugar a la Educación Intercultural Bilingüe. Por tanto, se toma en consideración que los recorridos de los proyectos de multiculturalismo e interculturalidad en Ecuador siempre remitirán especialmente a la historia de la educación y a las luchas de los movimientos indígenas.

Se reconoce que en Ecuador sucede lo mismo que para el indígena australiano Nakata:

(...) "el idioma de un pueblo y la historia de su desarrollo son todavía segundarios y siguen siendo una consideración subordinada a las interpretaciones de los lingüistas de cómo la gramática determina un significado (...)" (Nakata, 2014, p. 84).

Esto ha traído consecuencias nefastas como el desprestigio lingüístico y social de las lenguas ancestrales al no ser analizadas y consideradas por sus contextos de lucha, resignificación y necesidades de la propia educación, bajo el gran paraguas que ofrece el análisis del multiculturalismo y la interculturalidad desde una perspectiva crítica y funcional para los pueblos y las nacionalidades indígenas del Ecuador.

En este marco, ha sido fundamental reflexionar acerca de la urgencia de un giro epistemológico y crítico en la educación indígena e intercultural en Ecuador, tal y como lo definen autores del campo de los estudios de la cultura y de la comunicación en clave interdisciplinaria.

Por eso, este ensayo analiza qué es la Educación Intercultural Bilingüe y cuál ha sido el contexto teórico e histórico que incidió para su institucionalización, pasando por los procesos de resistencia como las luchas del movimiento indígena, hasta llegar a los recorridos por las ayudas internacionales y la participación de la religión y de un modelo de modernidad hegemónico.

Se coloca especial atención al marco legal y normativo nacional e internacional que ampara e impulsa los derechos por una EIB. Los documentos oficiales y la literatura académica colocan en contexto a la EIB en Ecuador para reconocer cuáles son los puntos de tensión que problematizan la situación de los Pueblos y Nacionales que reciben una EIB y cuál es la situación de la población hegemónica en Ecuador como lo es la sociedad blanco-mestiza.

Se reflexiona alrededor de construir cambios descolonizadores tomando en cuenta cuatro dimensiones que plantea De Souza Silva (2013): epistemológica, ontológica, metodológica y axiológica. En primer lugar, epistemológica en referencia a Walsh cuando reflexiona acerca de la incidencia del fenómeno del conocimiento y los procesos que lo construyen; ontológica en la medida en que se determinan unas realidades concretas capitalistas; metodológica porque se basa en los procesos de indagación a partir de métodos, y axiológica desde el tipo de intervención y los valores éticos y estéticos para el reconocimiento de la realidad.

Por último, se recomienda que las Políticas Públicas de Ecuador consoliden planes, programas y proyectos que respalden una relación más entre iguales en términos de alcanzar un diálogo intercultural para el desarrollo socio-cultural.

\section{Metodología}

El método aplicado es de revisión bibliográfica con teoría fundamentada que se contrastará con 
las principales aportaciones de la interculturalidad entendida de manera más compleja que una simple relación entre culturas. Por lo tanto, dentro de la selección de la bibliografía, destacan los estudios realizados por autores pertenecientes a las ciencias sociales y la comunicación bajo una perspectiva critica de la modernidad capitalista, para establecer relaciones radicalmente diferentes con formas de poder y vivir nuevas, lo que implica disciplinar y regular pensamientos y situaciones racializadas y excluidas. Por lo tanto, las disciplinas con las que principalmente se llevará el tema son: educación, estudios de la cultura y comunicación.

\section{Análisis y resultados}

Al estudiar la historia de la colonización nos conducimos hacia lo que Rodríguez (2017) explica a través de varios autores (Mariátegui, 1928; Cueva, 1980), que la situación de la educación indígena a partir de la consolidación del Estado-nación en América Latina se vio afectada por el capitalismo y el rechazo al indígena por considerarlo social, política y culturalmente, un ser inferior.

Tal es así, que para Vera Candau, desde la colonia hasta el siglo XX se consolida una etapa que tiene como marca "una violencia etnocéntrica explícita de imposición de la cultura hegemónica sobre las poblaciones indígenas. Eliminar al 'otro' fue la tónica del período colonial" (Vera, 2013 , p. 146). No obstante, de acuerdo con la autora, en la primera etapa del siglo XX incide el proyecto de Estado-nación moderno que devino en una tendencia asimilacionista, es decir en la estandarización y homogenización cultural y bajo esta mirada se construyeron las primeras instituciones educativas indígenas bilingües.

Mientras tanto, en el ámbito del desarrollo social en América, anterior a la década de los 50, debido a las influencias del occidentalismo y el capitalismo vigente, se buscaba el desarrollo como meta fundamental, es decir, como un punto de llegada. No obstante, en aquel tiempo se intentó que sea desde la iniciativa de los grupos no-hege- mónicos en donde países como Ecuador necesitaban "crecer hacia afuera" mediante la exportación para encontrar formas de prosperidad económica y social. Esto remite a la noción de que el indígena en un primer momento tuvo que revitalizar su cultura (multiculturalismo) motivado por políticas públicas estatales que deseaban mantener las desigualdades sociales, políticas, culturales y económicas y posteriormente el indígena tuvo que integrarse al resto de la sociedad para el diálogo (interculturalidad), lo que actualmente desde una mirada crítica y funcional se concibe como proyecto ético y político en construcción.

Con estos antecedentes, los marcos legales que se establecen para el análisis se han fundamentado. En el ámbito nacional, la base se encuentra en la Constitución Política de la República del Ecuador (2008) mientras que en lo internacional está el Convenio 169 de la OIT y los Derechos Humanos de los Pueblos Indígenas de las Naciones Unidas. Del documento magno nacional se desprenden varios documentos sobre la educación intercultural como los planes nacionales de Desarrollo que fueron publicados en distintos momentos. El último (2017-2023) corresponde al actual presidente, Lic. Lenín Moreno Garcés, entre otros documentos oficiales que como se verá, en Ecuador toman acción desde lo multicultural y no tanto desde lo intercultural.

La Constitución del Ecuador (2008) pasó por una serie de reformas, entre los cambios se incorporó un refuerzo a los derechos de los pueblos indígenas y afroecuatorianos. El artículo 68 (capítulo 4, sección octava) afirma que "el sistema nacional de educación incluirá programas de enseñanza conformes a la diversidad del país. Incorporará en su gestión estrategias de descentralización y desconcentración administrativas, financieras y pedagógicas."

Este artículo, permite tener en cuenta varias cosas. Primero, que el sistema de educación debe de incluir, por derecho, programas específicos estructurados según cada cultura. Segundo, que estos programas deben de ser manejados y ejecutados por personas de las 
comunidades correspondientes a cada cultura, ya que son ellos los que conocen su contexto y necesidades, razón por la cual el artículo 68 obliga al sistema educativo a desarrollar estrategias de descentralización y desconcentración. Tercero, este artículo obliga indirectamente a que los programas educativos sean desarrollados en la lengua de cada comunidad, ya que esta es un eje cultural esencial.

Según Bastidas, citando a Ferräo, desde los años 70 hasta los 80 se empezó a hablar de EIB en América Latina y específicamente en Ecuador se encuentra ligado a temas del Estado, de manera que desde una verticalidad se quiere definir lo étnico, sujetándolo a que esto es irrenunciable la lo ecuatoriano y ya no solo como un asunto que incumba únicamente a los indígenas (Bastidas, 2015).

De hecho durante la primera etapa de la educación indígena, en el contexto de las luchas de los movimientos sociales se encuentran Dolores Cacuango (1881-1971), Nela Martínez (19122004), María Luisa Gómez de la Torre (18871976), Tránsito Amaguaña (1909-2009). Así también, y gracias a la "Federación Ecuatoriana de Indios" (FEI) y por medio de otros grupos patrocinados por la "Alianza Femenina Ecuatoriana", se crean escuelas kichwa-español en Cayambe. $\mathrm{Al}$ ser esta una iniciativa que disgustaba a los hacendados, la educación a los niños se realizaba en horario nocturno y a escondidas.

Posteriormente se crearon programas educativos con el apoyo de la iglesia judeocristiana como el de las misioneras "Lauritas" de 1940 que estuvo a cargo de religiosas oriundas de Colombia que vivían en Ecuador. Y así, en años siguientes, toma iniciativa lo religioso en conjunto con el Ministerio de Educación. Más adelante en 1963 con el "Instituto Lingüístico de Verano" (ILV) se dieron a conocer las lenguas indígenas a cambio de difundir también la religión protestante a los grupos indígenas kichwas, shuar, secoyas, sionas, huaos, chachis y tsachis (Yánez, s.f.).

En el año 1979 durante el Gobierno del expresidente Jaime Roldós Aguilera, se institu- cionaliza con dificultades económicas y humanas, la Educación bilingüe intercultural donde destaca principalmente, en los años ochenta todo el proceso de lucha de los movimientos indígenas del Ecuador (Vélez, 2008; Moya, 1998).

En 1988 se crea la "Dirección Nacional de Educación Intercultural Bilingüe" (DINEIB). Esto constituyó una legitimidad a partir de la institucionalización, no obstante adicional a las problemáticas arriba mencionadas, se toma en cuenta la excesiva burocracia y mirada uninacional y vertical desde el Estado y la asimilación cultural (CONAIE, 1994, p. 44; Guerra, 2003).

En 1990, el movimiento indígena y en particular la "Confederación de Nacionalidades Indígenas del Ecuador" (CONAIE) exigió al Estado ecuatoriano que tomaran en cuenta sus derechos e intereses particulares en la educación de los niños indígenas (Abram, 2013; Moya, s.f.). Es entonces que se empieza a trabajar por un sistema de EIB propio, aunque no concluyente ni propiciador de la interculturalidad. Esta educación multiculturalista, intentaba revitalizar a la propia cultura, sin embargo, muchas veces los mismos docentes, estudiantes y padres de familia estuvieron en desacuerdo con el tipo de educación (Abram, 2013, pp. 2-3).

Se podría revisar cómo la EIB desde sus inicios hasta los años noventa del siglo XX buscó el desarrollo de unas políticas públicas afianzadas en el multiculturalismo, por el fortalecimiento de la propia cultura, especialmente desde lo lingüístico. Fue parte de la agenda pública, debido a la presión de los movimientos indígenas, así como también por la participación religiosa y de organismos extranjeros privados y desde el Estado.

A pesar de los grandes esfuerzos del Gobierno del Ecuador para visibilizar y empoderar a la comunidad indígena a través de la Constitución con énfasis en la educación intercultural bilingüe, el último censo del año 2010 evidenció que el $20,4 \%$ de la población indígena se auto identificaba como analfabeta. Si bien durante dicho año se muestra que hubo un incremento en la escolaridad de educación bási- 
ca y bachillerato de hombres y mujeres, la población indígena seguía marginada (INEC, 2010).

Por esa razón en el 2011 el Ministerio de Educación ofició el "Proyecto de fortalecimiento de la educación intercultural bilingüe" con cuatro años de duración. Este proyecto estaba dirigido a catorce nacionalidades en veintidós provincias del Ecuador y tenía como objetivo mejorar la calidad del programa de Educación Intercultural Bilingüe (Ministerio de Educación, 2010). No obstante, el Ministerio señaló que el principal problema relacionado con el proyecto radicó en "la baja calidad de los servicios educativos de los pueblos y nacionalidades que garantice la permanencia de las lenguas y las culturas" (2010). Debido a ello, el Ministerio de Educación capacitó a docentes en técnicas pedagógicas, material didáctico para alumnos que no han adquirido el kichwa como lengua materna y validación mediante la incorporación de las necesidades de cada nacionalidad indígena en los currículos.

El Plan Nacional del Buen Vivir (20092013) fue creado durante el Gobierno del expresidente, economista Rafael Correa Delgado, y derivado del principio ancestral de Sumak Kawsay, que conceptualiza y promete para Ecuador en términos de Desarrollo, mejoramientos en lo político, económico, social, cultural y ecológico. Un instrumento para conjugar las políticas públicas con la administración del Gobierno y la inversión pública.

En el Plan Nacional Buen Vivir (20132017) de los doce objetivos, el 2 y el 4 respaldaron el Sistema de Educación Intercultural Bilingüe. El Objetivo 2 garantiza la igualdad a través del acceso a servicios de salud y educación de calidad a personas y grupos que necesitan especial atención por la permanente desigualdad, exclusión y discriminación. Entre sus objetivos se plantea "fortalecer y concentrar los programas de alfabetización y postalfabetización para aquellas personas con escolaridad sin terminar, desde un punto de vista en que se cierren las brechas, con base a la identidad de género, cultural y territorial". La meta 2.5 de este objetivo plantea incentivar la inclusión y cohesión social, la convivencia pacífica y la cultura de paz, eliminado todas las formas de discriminación y violencia. En específico, "crear mecanismos de comunicación y educativos que promuevan el respeto y el reconocimiento de la diversidad y afirmen el diálogo intercultural y el ejercicio de los derechos colectivos de las nacionalidades y los pueblos indígenas". Por su parte, el objetivo 4 del Buen Vivir 2013-2017 está centrado en fortalecer las capacidades y potenciales de la ciudadanía, el cual busca garantizar a todos los ciudadanos su derecho a la educación, bajo el precepto de equidad y la sustentabilidad. Todas las metas de este objetivo giran en torno a la educación.

Siguiendo a estos objetivos, dentro del Sistema de Educación Intercultural Bilingüe se han registrado 46588 estudiantes y se han capacitado a 4406 docentes en el 2012 (Buen Vivir, 2013). Según datos de la Secretaría Nacional de Planificación y Desarrollo, el Plan de desarrollo ha tenido un alcance positivo durante el primer año, ya que en la educación básica superior se observó un incremento de tres puntos porcentuales entre 2012 y 2013, al igual que la tasa de bachillerato subió en dos puntos dentro del mismo periodo. Otro indicador positivo muestra que el analfabetismo en la población indígena ( 15 a 49 años), pasó del 11\% en 2012 a $7.6 \%$ en 2013. Sin embargo, en secundaria hubo deserción escolar del 8.7\%. Uno de los principales motivos del abandono escolar en esta etapa, muestra que el $41 \%$ adquiere responsabilidades económicas desde muy jóvenes por lo tanto deben trabajar a una edad temprana, el $18 \%$ por falta de recursos económicos, el $13 \%$ por atender los quehaceres del hogar y el 11\% porque no estaba interesado.

En el actual Gobierno del Licenciado Lenín Moreno Garcés, se considera en el último Plan Nacional de desarrollo (2017-2023) que el Estado tiene tres obligaciones neurálgicas que son determinantes para su correcto funcionamiento: respetar, proteger y realizar. Respetar que el Estado no vulnere los derechos y proteger 
para evitar que aquello suceda (y en caso de que ocurra exigir las reparaciones del caso) y por último, realizar implica que el Estado debe ser proactivo para garantizar los derechos, con énfasis a grupos de atención prioritaria. En este sentido, la Constitución de la República del Ecuador (2008) en el artículo 57, núm. 14 señala que en la Educación superior se debe potenciar el Modelo del Sistema de Educación Intercultural Bilingüe (MOSEIB) oficializado en 1993, con última edición del documento presentada en el 2013, y que los principales actores sociales del Sistema de EIB deben ser los mismos pueblos y nacionalidades históricamente oprimidos.

Por último, se puede señalar que este Plan de Desarrollo, elaborado durante el Gobierno del actual presidente de la República del Ecuador, igual que los dos anteriores documentos, señalan la relevancia de ajustar la Educación Intercultural Bilingüe con:

Pertinencia cultural, lingüística y ambiental, que atienda a las necesidades educativas específicas de los pueblos y nacionalidades. Esto implica revalorizar la educación intercultural bilingüe como una conquista histórica de los pueblos y nacionalidades, y destacar su reivindicación intergeneracional al resistir procesos de homogenización y asimilación.

Así, la educación intercultural bilingüe y la etnoeducación encasillan y se mantienen con el actual Gobierno, lo mismo que con el anterior presidente del economista Rafael Correa Delgado, al menos en términos discursivos, como prioridades permanentes en la educación del país, desde la estimulación temprana hasta la educación superior.

Los organismos internacionales también han tenido influencia en las decisiones gubernamentales para el desarrollo de la población indígena. En 1989, el Ecuador firmó con la Organización Mundial del Trabajo (OIT) el convenio de Pueblos Indígenas y Tribales. Dicho convenio tiene cuatro ejes principales: el derecho de los pueblos indígenas a mantener sus costum- bres, fortalecer sus culturas, defender sus diferentes formas de vida y su derecho a participar de manera activa en las decisiones que le atañen (OIT, 1989). Lo mencionado establece las bases en la cual se debe interpretar las disposiciones del convenio.

En la parte quinta de seguridad social, el artículo 27 establece que:

Los programas y los servicios de educación destinado a los pueblos interesados, deberán desarrollarse y aplicarse en cooperación con éstos a fin de responder a sus necesidades particulares, y deberán abarcar su historia, sus conocimientos y técnicas, sus sistemas de valores y todas sus demás aspiraciones sociales, económicas y culturales. (OIT, 1989)

A través de este artículo, Ecuador promete una vez más, autonomía a las comunidades indígenas para desarrollar sus propios programas educativos con el apoyo del estado.

En el literal 2 del mismo artículo se establece que la autoridad (...) "deberá asegurar la formación de miembros de estos pueblos y su participación en la formulación y ejecución de programas de educación, con miras a transferir progresivamente a dichos pueblos la responsabilidad de la realización de esos programas, cuando haya lugar" (OIT, 1989). Este literal obliga al estado a preparar a miembros de estas comunidades en materias educativas para que en el futuro estas comunidades, en total autonomía, desarrollen sus propios programas educativos. Además, el artículo 27 sugiere que instituciones públicas se relacionen e integren con las comunidades para conocer de cerca la realidad social, cultural, lingüística y desarrollar los programas educativos concernientes (Conejo Arellano, 2008).

Por su parte, el "Programa de Desarrollo y Diversidad Cultural para la Reducción de Pobreza e Inclusión Social" (PDC) se llevó a cabo mediante el cumplimiento de los Objetivos del Desarrollo Sostenible, planteados por las Naciones Unidas. De los diecisiete objetivos, el cuarto busca "garantizar una educación inclusiva 
y equitativa de calidad y promover oportunidades de aprendizaje permanente para todos" (Naciones Unidas, 2016). Por lo tanto, se pretende que toda la población tenga acceso pleno y digno al trabajo, a la educación y a la salud.

Cabe mencionar que según el "Informe Sobre Desarrollo Humano" de 2016, los pueblos indígenas representan el $5 \%$ de la población mundial, sin embargo, el 15\% son de escasos recursos económicos (Naciones Unidas, 2017). Teniendo en cuenta esta problemática, se elaboró como política pública el PDC para erradicar la discriminación racial y la exclusión étnica y cultural el 28 de diciembre del 2009. Parte de dicha política pública buscó desarrollar la educación inicial indígena en la que se elaboraron cuatro diccionarios de las nacionalidades Secoya, Cofanes, Wao y Shuar. A su vez, el Ministerio de Salud programó un cambio en el sistema de salud para entregar un servicio adecuado a las necesidades de la población indígena (Ministerio de Cultura y Patrimonio, 2016).

El emprendimiento también fue un pilar importante para sacar adelante a la población indígena, por lo que se creó un plan de sostenibilidad en varios sectores, en pro de la recuperación de los conocimientos ancestrales (Ministerio de Cultura y Patrimonio, 2016). Debido al éxito del proyecto el Gobierno realizó una segunda fase del PDC cuyo objetivo fue ayudar a reforzar a la sociedad intercultural mediante espacios de reflexión, marcos normativos e institucionales y la creación de políticas públicas para realzar la cultura, el diálogo intercultural, y disminuir los espacios de discriminación hacia los pueblos y nacionalidades que viven en el Ecuador (Ministerio de Cultura y Patrimonio, 2016). Los resultados de este proyecto entre el año 2013 y el 2015 fueron positivos ya que 16 de los 28 emprendimientos de la comunidad indígena tienen planes de negocios, que les ha ayudado a posicionar sus productos en mercados locales fomentando la economía en lugares lejanos, no obstante se instrumentaliza al indígena insertándolo al mercado capitalista. Así también, se ha ampliado la oferta de productos artesanales, turísticos, agrícolas y pecuarios en los mercados locales y cantonales (Ministerio de Cultura y Patrimonio, 2016). Por último, el emprendimiento permitió devolver y fortalecer las costumbres, tradiciones y saberes ancestrales comunidades indígenas pero no necesariamente a construir una hegemonía.

Por otra parte, es imperante evidenciar el Acuerdo del Ministerio de Educación donde se establece que los currículos de "Educación General Básica" para los procesos de "Educación Infantil Familiar Comunitaria" (EIFC), contemple una asimilación cultural desde la "Inserción a los Procesos Semióticos (IPS), Fortalecimiento Cognitivo, Afectivo y Psicomotriz (FCAP), Desarrollo de Destrezas y Técnicas de Estudio (DDTE) y Proceso de Aprendizaje Investigativo (PAI)" (artículo 2). Lo indicado, sin el direccionamiento necesario pudiera ser asumido en la verticalidad y la falta articulación de un diálogo que permita la comunicación horizontal entre los pueblos y nacionalidades del Ecuador. No obstante pensando en modelos de aplicación prestados por sistemas occidentales en el ámbito educativo, esto a pesar de que el acuerdo entre las disposiciones generales del sexto apartado, se indica que el Estado debe "Responsabilizar a los docentes y autoridades de los establecimientos educativos interculturales bilingües, de la generación de mecanismos permanentes de investigación, construcción colectiva de recursos educativos con pertinencia cultural y lingüística" todo esto con pertinencia a las relaciones entre culturas (MinEdu, 2017, p. 5).

El campo de las relaciones entre culturas ha sido complejo de definir, pues esta carga con una serie de representaciones y sentidos. Se toma en cuenta el concepto interculturalidad a partir de las reflexiones de Catherine Walsh (2001, 2005,2008 ), quien ha señalado que tal noción se asienta en la necesidad de la transformación radical de las estructuras, instituciones y relaciones de la sociedad. Señala la autora, que la interculturalidad es eje central de un proyecto histórico 
alternativo. Walsh irá más allá y explicará que la interculturalidad —entendida como herramienta y proyecto necesario para la transformación trascendental del Estado y la sociedad - necesita romper con el marco uni-nacional, recalcando lo plura-nacional no como división, sino como estructura más adecuada para unificar e integrar.

Por ello se afirma que el campo de las relaciones entre culturas ha sido complejo de definir. Más aún, la problemática radica en traducirlo en términos de una política pública transformativa. Conviene tener presente que la dificultad empieza cuando en una sociedad particular predomina una clase que, bajo el halo de supremacía cultural, tiene mejores condiciones de vida en su contexto; tal disyuntiva predispone al reconocimiento y valoración de las minorías culturales que terminarán siendo marginalizadas bajo las manipulaciones del orden hegemónico implícitos.

Por eso vale precisar las diferencias entre multiculturalismo e interculturalidad. Por una parte, el multiculturalismo responde a la 'esencia' de las culturas, el interculturalismo enfatiza la interacción o dinámicas entre al menos dos culturas, sin desconocer los escenarios de disputa de poder, vínculos, negociaciones, cooperación y conflicto (González, 2005).

En este sentido, resultará útil tener en cuenta las reflexiones que ha desarrollado Virgilio Alvarado en el marco de la interculturalidad y las políticas públicas. Dicho autor señala que la propuesta de la interculturalidad aspira a una sociedad intercultural amparada en un proyecto político que permita establecer un diálogo entre culturas; este dialogo, señala el autor, debe partir de la aceptación de la propia identidad y de la autoestima (Alvarado, 2002).

\section{Discusión y conclusiones}

Siguiendo una limitada revisión antropológica de la educación de los pueblos originarios de Ecuador contrastada con otras realidades de América, en Bolivia y en el propio país ecuatoriano, destacan las luchas de los movimientos indígenas y también el apoyo de organismos internacionales. En cambio, en Perú el apoyo fue posible gracias al Estado y a ciertos proyectos experimentales que sucedieron. Sea como fueran los procesos que en distintos países se han llevado a cabo, en la actualidad se ha apostado por estrechar las relaciones con la comunidad correspondiente a cada geografía, de tal suerte que se inmiscuya en proyectos de orden intercultural (Zavala, 2007).

En Ecuador convergen múltiples culturas y la multiculturalidad es una realidad pero la interculturalidad deviene en proyecto por construir. Es decir, dada la multiculturalidad no quiere decir que las culturas convivan en armonía pues el indígena se encuentra en franca desventaja debido al desprestigio cultural histórico. Tal es así que en la cultura kichwa se extienden expresiones como "runa shimi” y "yanka shimi” que quiere decir "la lengua que no vale" (Abram, 2013). Esta transformación de la lengua ancestral demuestra la necesidad de una revitalización cultural, lo que merece otros esfuerzos descolonizadores, reconociendo que la interculturalidad es el motor fundamental para el desarrollo y la globalización podría empujar a aplicar un modelo funcional a las realidades y contextos socio-culturales.

Por eso, se propone seguir la metáfora de la ecología de los medios (Scolari, 2015) para hacer una propuesta comunicacional que permita incluir al indígena en un mundo globalizado y así construir otras representaciones de lo indígena. Estas transformaciones deben suceder en el campo de la comunicación análoga y digital siguiendo estrategias de comunicación que permitan la evolución y la transformación socio-cultural de los seres racializados, y principalmente desde el campo de la educación se pueden llevar estos esfuerzos, caso contrario y siguiendo la metáfora de Scolari (citando a McLuhan), las culturas ancestrales podrían caer en peligro de extinción.

En el marco de la siguiente propuesta lo importante es reconocer las similitudes culturales (comunicar, es acercar, poner el común, 
comulgar) con realidades coexistentes de un país multicultural como Ecuador pero dando pasos concretos para construir la interculturalidad.

La realidad siempre que trascienda de un monólogo a un diálogo y comunicación horizontal, compromete a la interculturalidad entendiendo ésta como políticas públicas para el diálogo entre culturas y eso exige la participación de los seres racializados y de la población blanco-mestiza.

Varios autores coinciden, cuando se refieren a que la interculturalidad es un proyecto en construcción, en tanto se asumiría que el único intercultural de esta dualidad es el indígena y contadas veces el blanco mestizo quien entraría en intentos de diálogo con el otro (socialmente asumido como contrario y diferente).

Así, la interculturalidad en Ecuador no es un hecho dado (como refiere el concepto de multiculturalidad), sino que al ser un proyecto, debe llevarse a cabo mediante una planificación a largo plazo que deslinde las afectaciones de la colonización a más de quinientos años, no obstante, no ha sido prioridad de la agenda del Gobierno en materia de políticas públicas. Lo recomendable sería dar un soporte a la revitalización cultural del Ecuador, y eso implica apostar a un cambio de la matriz productiva y a un cambio hegemónico, para que luego sea considerado una prioridad gubernamental, no obstante, al mismo tiempo que se empiece a considerar que entre las lenguas oficiales del país, de acuerdo con la Constitución (2008), se encuentran el kichwa y el shuar, entonces, se espera empezar esta tarea de enseñar estas lenguas en instituciones públicas y privadas para así garantizar que una vez más que los únicos interculturales no sean los seres racializados. Deben por lo tanto, coexistir otras formas de gestión del conocimiento, para aprender y convivir en espacios de interculturalidad propiciados por el mestizaje social y cultural.

La llegada de los conquistadores a América dio lugar a una serie de conflictos que marcó la dicotomía y segregación de los grupos indígenas del país, por eso es complejo escapar de esas trampas de racionalización donde se mira al otro como un subdesarrollado o un salvaje.

Se considera neurálgico desarrollar e impulsar desde la comunicación, una agenda política y militante para el diálogo intercultural, por ello los medios de comunicación y las agencias de publicidad tienen un rol fundamental como productores de sentidos sociales. Además, no sería un retroceso destacar la propuesta del multiculturalismo para extender aún más la lengua kichwa en Ecuador, a sabiendas de que con este aprendizaje se pueden entender, valorar y revitalizar a las cultura Otras.

Se recomienda buscar nuevas formas que desde la comunicación de campañas sociales se concientice en los usos sociales de términos en kichwa como "runa" cuyo significado literal es persona, identidad. Además, es conveniente que los blancos-mestizos abracen su cultura ecuatoriana fragmentada y minimizada que históricamente ha sufrido discriminación, se deben buscar nuevos horizontes epistemológicos y empíricos para la construcción de identidades sociales. El desafío está en el reconocimiento igualitario en un contexto globalizado para posibilitar la integración social en clave intercultural.

En suma, la interculturalidad no puede — no debe- ser asumida como una categoría de análisis que omita la disposición al diálogo con la diferencia cultural. Por lo tanto, toma particular importancia el planteamiento de políticas públicas que definan la participación de los blancomestizos de manera que exista una comunicación y un diálogo integral, dejando de lado las actividades de folclorización cultural y formas del monoculturalismo de herencia occidental.

\section{Referencias bibliográficas}

Abram, M. (2013) La Educación Bilingüe Intercultural en las cuatro nacionalidades de la costa del Ecuador. Quito: Prometeo Senescyt.

Conejo Arellano, A. (2008). Educación Intercultural Bilingüe en el Ecuador. En La propuesta educativa y su proceso. (https://bit.ly/2Q6bnpP) 
Bastidas Jiménez, M. (2015). Educación Intercultural Bilingüe en el Ecuador: Un estudio de la demanda social. Alteridad, 10(2), 180-189.

Confederación de Nacionalidades Indígenas del Ecuador -CONAIE (1994). Proyecto político de la CONAIE. Quito: Consejo de Gobierno de la CONAIE.

Constitución Política de la República del Ecuador (2008).

De Souza Silva, J. (2013). La pedagogía de la felicidad en una educación para la vida. El paradigma del 'buen vivir' / 'vivir bien' y la construcción pedagógica del 'día después del desarrollo'. En C. Walsh (Ed.), Pedagogías decoloniales. Prácticas insurgentes de resistir, (re) existir y (re) vivir. Quito: Abya-Yala.

González, I. (2005). La cooperación educativa ante la rebeldía de las culturas (1ra. ed.). España: Editorial IEPALA.

Guerra, F., (2003) VI. Las mutaciones de la identidad en la América Hispánica. En Antonio Annino, François-Xavier Guerra (Coords.), Inventando la nación. Iberoamérica siglo XIX (pp.185-220) México: FCE.

INEC (2010). El Censo informa: Educación. (https://bit.ly/1K4kb3v).

Ley Orgánica de Educación Intercultural (LOEI) (2011). (https://bit.ly/2W2PyNp) (2018/01/17).

Ministerio de Cultura y Patrimonio (2016). Proyecto Desarrollo de y Diversidad Cultural para la Reducción de la Pobreza e inclusión social, PDC. Segunda Fase (2013-2016). (https://bit.ly/2VwPJvY)

Ministerio de Educación (2013). Modelo del Sistema de Educación Intercultural Bilingüe (MOSEIB). (https://bit.ly/2C6vkWO).

Moya, R. (1998). Reformas educativas e interculturalidad en América Latina, s.c., Revista Iberoamericana de Educación. Número 17. Educación, Lenguas, Culturas.
Naciones Unidas (2016). Informe de los Objetivos de Desarrollo Sostenible. (https://bit.ly/2sPPdvi).

Naciones Unidas (2017). Informe Sobre Desarrollo Humano 2016. (https://bit.ly/2nezplJ).

Nakata, M. (2014). Disciplinar a los salvajes, violentar las disciplinas. Quito: Abya-Yala.

OIT (1991). Convenio 169 de la Organización Internacional del Trabajo.

Rodríguez Caguana, A. (2017) El largo camino del Taki unkuy. Los derechos lingüísticos y culturales de los pueblos indígenas del Ecuador. Quito: Huaponi Ediciones.

Scolari, C. (2015). Ecología de los medios. Entornos, evoluciones e interpretaciones. Barcelona: Gedisa. Senplades (2013). Plan NacionaldeDesarrollo, Buen Vivir. (https://bit.ly/2nezplJ).

Senplades (2017) Plan Nacional de Desarrollo, Toda Una Vida. (https://bit.ly/2hrQrcp).

Vélez, C. (2008). Trayectoria de la Educación Intercultural en Ecuador. Revista Educación y Pedagogía, 103-112. Buenos Aires. (https://bit.ly/2VKibiU) (2017/01/02).

Vera Candau, M. (2013). Educación Intercultural crítica. Construyendo caminos. En Pedagogías decoloniales. Prácticas insurgentes de resistir, (re) existir y (re) vivir. Quito: Abya-Yala.

Walsh, C. (2001). La Educación Intercultural en la Educación. Lima: Ministerio de Educación.

Walsh, C. (2005). Pensamiento crítico y matriz (de) colonial. Reflexiones latinoamericanas. Quito: Editorial Abya-Yala.

Walsh, C. (2008) Interculturalidad y plurinacionalidad: Elementos para el debate constituyente. Quito: Universidad Andina Simón Bolívar.

Yánez Cossío, C. (s.f.) "Macac": Teoría y práctica de la educación indígena. Estudio de caso en el Ecuador. Quito: Macac.

Zavala, V. (2007). Avances y desafíos de la educación intercultural bilingüe en Bolivia, Ecuador y Perú. Estudio de casos. Perú: CARE. 